\title{
Enlightened engineering
}

\author{
Optogenetics—until now primarily a tool for asking questions in basic research—is starting to spur efforts oriented \\ toward biomedical applications.
}

O ptogenetics- the use of light to control cellular activities-has proven a tremendous research tool in neuroscience. Although only a few applications outside of basic research have emerged as yet, several papers in the past eighteen months provide tantalizing glimpses of the potential of light-activated receptors and channels in biomedicine. This illustrates how an area of curiosity-driven research can yield unanticipated benefits for biomedical technology.

Although pioneered in the laboratory of Gero Miesenböck, optogenetics first attracted wide attention in 2005 when Karl Deisseroth, Edward Boyden and colleagues demonstrated that an opsin from green algae could render neurons responsive to light. Over subsequent years, the expression of photoswitchable proteins in the brain of living miceeven conscious, freely moving animals-has become a key experimental approach in neuroscience. Excitatory optical switches like blue lightactivated channelrhodopsin have been joined by inhibitory photoactive proteins (e.g., the yellow light-activated halorhodopsin chloride ion pump), opening up the possibility of combining different opsins and their variants with different 'effector' domains.

Current optogenetic probes remain a work in progress. Absorption spectra for many photoresponsive receptors and channels lie in the blue and yellow range, limiting the depth at which activation can be achieved in tissues. Activation spectra are often broad and overlapping, making the simultaneous use of different probes and fluorescent reporters difficult. Most light-activated microbial proteins permit millisecond-precision optical control of engineered cells, but the kinetics and dynamic range of opsins are an area of intense investigation as is the search for switches that are less leaky in the 'off' state or show higher levels of activation when 'on'.

Ideally, protein engineers would prefer generalizable switches that can be applied to many different proteins for different situations. Promising approaches include fusions with LOV (light, oxygen, voltage) domains, which undergo large structural changes upon illumination, or dimerization domains that can bring proteins to a desired subcellular location or induce protein-protein interactions for a desired activity. It is also possible that sequencing of microbial communities will yield new opsins.

Given that optogenetics has been around as a research tool for just five years, it is perhaps surprising that it has already yielded several areas of promising translational research.

Most work has focused on neurological applications. For example, optogenetics has been used to investigate the role of afferent axons, excitatory neurons and astroglia within the subthalamic nucleus as well as layer V motor cortex projection neurons in Parkinson's models. Similar work has been carried out in depression and anxiety disorders.

Ultimately, it is hoped that a combination of viral optogenetic therapy and optical-fiber implants could enable treatments to combat specific neural abnormalities in neurodegenerative disease. To some, the prospect of patients walking around with holes drilled in their skulls and optical fibers in their heads appears remote. But wirelessly powered LED light devices weighing only about $2 \mathrm{~g}$ are already in the pipeline (J. Neural Eng. 8, 046021, 2011). And such treatments are not so unrealistic when one considers a substantial number $(\sim 70,000)$ of Parkinson's patients already opt for deep brain stimulation (DBS), an invasive procedure in which electrodes are implanted deep inside the brain.

One particularly compelling application of optogenetic therapy is in eye diseases where photoreceptor cells have died or are compromised but the retinal circuitry remains largely intact. Several groups, including startup Eos Neuroscience, have shown in blind model mice that adeno-associated virusmediated delivery of opsins to retinal cells leads to electrophysiological and behavioral improvements in sight, with visual responses lasting in some cases as long as 10 months.

In the area of neuroprosthetics, optogenetic stimulation of motor neurons might also have advantages. Devices using electrical stimulation tend to preferentially recruit only large bundles of muscle fibers, leading to rapid muscle fatigue and compromised muscle control and force. When motor neurons are instead stimulated with channelrhodopsin and light, the normal order of muscle fiber recruitment is preserved, avoiding such problems. This unanticipated finding has suggested new possibilities for neuroprosthetic devices. In similar work, a startup called LucCell is using vertebrate rhodopsin 4 in mouse models to turn off the urethral sphincter muscle with a view to restoring bladder control.

There have also been papers describing the expression of channelrhodopsin in the hearts of transgenic mice, enabling precise manipulation of electrical activity with high spatial and temporal resolution. This hints that optogenetic pacemakers may one day find a niche in such conditions as atrial fibrillation, which confound existing pacemaker technology. And in the area of metabolic disease, optical control of glucose homeostasis has recently been demonstrated by means of a cellular signal transduction cascade based on melanopsin that ultimately leads to expression of recombinant peptide glucagon-like peptide 1 (GLP-1) (Science 332, 1565-1568, 2011). Diabetic mice implanted with encapsulated cells engineered to express melanopsin and GLP-1 are capable of controlling glucose levels in response to light.

Of course, all of these applications lie many years away from the clinic. Human optogenetics will require regulatory concerns about vector toxicity, delivery efficiency, cell targeting specificity, immunogenicity and insertional mutagenesis to be addressed and studies set up to ascertain whether long-term expression of foreign opsins elicits immune responses. Approaches requiring photo-stimulatory devices as well as gene therapy will add to the regulatory complexity.

In the meantime, optogenetics will continue to find new uses in basic research, even in screens to identify drugs that act on specific neural cell types. Who would have thought that a simple protein from a lowly alga would light up so many avenues in translational research? 